\title{
The Equivalence of Two Graph Polynomials And a Symmetric Function
}

\author{
Criel Merino* \\ Instituto de Matemáticas \\ Universidad Nacional Autónoma de México \\ Area de la investigación científica \\ Circuito Exterior, C.U. Coyoacán 04510, \\ México, D.F. México. \\ Steven D. Noble ${ }^{\dagger}$ \\ Department of Mathematical Sciences \\ Brunel University \\ Kingston Lane \\ Uxbridge \\ UB8 3PH \\ United Kingdom
}

November 12,2018

\begin{abstract}
The $U$-polynomial, the polychromate and the symmetric function generalization of the Tutte polynomial due to Stanley are known to be equivalent in the sense that the coefficients of any one of them can be obtained as a function of the coefficients of any other. The definition of each of these functions suggests a natural way in which to generalize them which also captures Tutte's universal $V$-functions as a specialization. We show that the equivalence remains true for the extended functions thus answering a question raised by Dominic Welsh.
\end{abstract}

\section{Introduction}

This paper answers a question posed by Dominic Welsh in a talk in 2005 [17 concerning the notions of equivalence and specialization of graph polynomials

* Supported by CONACYT of México.

†Partially supported by the Heilbronn Institute for Mathematical Research, Bristol, U.K. and by the Royal Society. 
and symmetric functions. We say that for graph polynomials $P$ and $Q, P$ specializes to $Q$ written $P \succ Q$ if the coefficients of $Q$ may be obtained as functions of the coefficients of $P$ and the number of vertices of the graph. Graph polynomials $P$ and $Q$ are equivalent if $P \succ Q$ and $Q \succ P$. These notions may be extended to symmetric functions by allowing the coefficients to be those of a symmetric function with respect to some basis. Defining equivalence in the right way is not completely straightforward [9], but this very simplistic notion will suffice for our purposes. Equivalence of graph polynomials or symmetric functions is clearly an equivalence relation.

Many specializations of the Tutte polynomial are well-known and include the chromatic and reliability polynomials. The key objects in this paper are extensions of two graph polynomials and one symmetric function that themselves generalize the Tutte polynomial. Brylawski introduced the polychromate [2], which is a polynomial in countably infinitely many variables. Stanley generalized the definition of the chromatic polynomial [13] to the chromatic symmetric function and a little later extended this to a symmetric function generalization of the Tutte polynomial [14]. For brevity we call this the Tutte symmetric function. Motivated by problems from knot theory, Noble and Welsh introduced the $U$-polynomial [10] and proved that it is equivalent to the Tutte symmetric function. Sarmiento 12 then showed that the $U$-polynomial and the polychromate are equivalent.

Taking for the moment an entirely naive and informal perspective, the definitions of these three functions lack a certain symmetry. In each of them the $x$ variable of the Tutte polynomial is generalized to countably infinitely many variables whereas the $y$ variable remains essentially unchanged. More importantly none of the three functions specializes to Tutte's universal $V$-functions 15 . It turns out that there are natural ways to define extended versions of each of these polynomials to overcome this problem which at the same time address the lack of symmetry.

The question asked by Welsh [17] was whether the equivalence of the $U$ polynomial and the polychromate carries over to their extended versions. Sarmiento's proof is quite involved and extending her methods did not appear to be an easy task. A key step in our approach is to introduce an extension of the Tutte symmetric function as an intermediate object between the two polynomials. Our main results are that the extended Tutte symmetric function is equivalent to both the extended polychromate and the extended $U$-polynomial. Since equivalence is transitive this answers Welsh's question. Our proof suggests a way to simplify Sarmiento's proof.

An overview of the paper is as follows. In the next section we present some preliminary definitions concerning symmetric functions. We then define the previously studied polynomials covered here and briefly survey some of their properties. Section four contains the definitions of two new graph polynomials and one new graph symmetric function together with our main results. We end with a brief conclusion and an open problem. 


\section{Partitions and symmetric functions}

We give some definitions and fix some notation which we will use throughout the paper. Note that all of our graphs are finite and may have multiple edges and loops. When the underlying graph is obvious we use $V$ and $E$ to denote respectively its set of vertices and edges and let $n=|V|$ and $m=|E|$. Given a graph $G, G \mid A$ is formed by deleting all the edges in $E \backslash A$ (but keeping all the vertices). We use $k(G)$ to denote the number of connected components of $G$ and define the rank of a set $A$ of edges to be given by $r(A)=|V|-k(G \mid A)$. If $A \subseteq E$ then let $\pi(A)$ denote the partition of $V$ for which the blocks are the connected components of $G \mid A$.

Given a partition $\pi$ of a set $A$, its type is the integer partition of $|A|$ for which the parts are the sizes of the blocks of $\pi$. If $\tau$ is an integer partition of $n$, we write $\tau \vdash n$ and let $k(\tau)$ be the number of parts of $\tau$. As usual we write the components of an integer partition in decreasing order so that if $\tau=\left(n_{1}, n_{2}, \ldots, n_{k}\right)$, we have $n_{1} \geq n_{2} \geq \ldots \geq n_{k}$.

We next introduce two symmetric function bases. For $r \geq 1$ let

$$
p_{r}(\mathbf{x})=p_{r}\left(x_{1}, x_{2}, \ldots\right)=\sum_{i=1}^{\infty} x_{i}^{r} .
$$

Now suppose that $\tau=\left(n_{1}, \ldots, n_{k}\right)$ is an integer partition of $n$. Then we define $p_{\tau}(\mathbf{x})$ to be the symmetric function $\prod_{i=1}^{k} p_{n_{i}}(\mathbf{x})$. The collection $\left\{p_{\tau}(\mathbf{x}): \tau \vdash\right.$ $n\}$ forms a basis called the power-sum basis for the homogenous symmetric functions of degree $n$ in $\mathbf{x}[8$.

Elements of the second basis, the augmented monomial basis are also defined in terms of an integer partition $\tau=\left(n_{1}, \ldots, n_{k}\right)$ of $n$. Let

$$
m_{\tau}(\mathbf{x})=\sum_{\left(i_{1}, \ldots, i_{k}\right)} x_{i_{1}}^{n_{1}} \cdots x_{i_{k}}^{n_{k}}
$$

where the sum is over all $k$-tuples of pairwise distinct strictly positive integers. Again, the collection $\left\{m_{\tau}(\mathbf{x}): \tau \vdash n\right\}$ is a basis for the homogenous symmetric functions of degree $n$ in $\mathbf{x}$. Note that $m_{\tau}$ is often denoted by $\tilde{m}_{\tau}$.

We now generalize some of these ideas to what we call paired symmetric functions. We have not been able to find any reference to these objects in the literature but surely they have been encountered many times before. First we define an integer pair partition of a pair of strictly positive integers $(a, b)$ to be a list of pairs of integers $\left(\left(a_{1}, b_{1}\right), \ldots,\left(a_{k}, b_{k}\right)\right)$ such that

1. $\left(a_{1}, \ldots, a_{k}\right)$ is an integer partition of $a$;

2. for all $i, b_{i}$ is a non-negative integer and $\sum_{i=1}^{k} b_{i} \leq b$;

3. if $i<j$ then either $a_{i}>a_{j}$ or $a_{i}=a_{j}$ and $b_{i} \geq b_{j}$, that is the pairs $\left(a_{i}, b_{i}\right)$ are written in lexicographically decreasing order. 
If $\bar{\tau}$ is an integer pair partition of $(a, b)$, we write $\bar{\tau} \vdash(a, b)$.

The canonical example of an integer pair partition is as follows. For a graph $G$, let $\pi$ be a partition of its vertex set. Order the blocks of $\pi$ in an arbitrary way. Let $a_{i}$ denote the number of vertices in the $i$ th block and let $b_{i}$ denote the number of edges of $G$ having both endpoints in the $i$ th block. Now write the pairs $\left(a_{i}, b_{i}\right)$ in lexicographically decreasing order to obtain the integer pair partition which we denote $\bar{\tau}(\pi)$.

We next define a paired symmetric function. Suppose $f$ is a function in the pairs of variables $\left(x_{1}, t_{1}\right), \ldots,\left(x_{i}, t_{i}\right), \ldots$ such that for any permutation $\pi$ of $\mathbb{Z}^{>0}$

$$
f\left(\left(x_{\pi(1)}, t_{\pi(1)}\right), \ldots,\left(x_{\pi(i)}, t_{\pi(i)}\right), \ldots\right)=f\left(\left(x_{1}, t_{1}\right), \ldots,\left(x_{i}, t_{i}\right), \ldots\right) .
$$

We require additionally that $f$ is homogenous in the $\mathbf{x}$ variables. Then we call $f$ a paired symmetric function. Notice that $f$ is not generally a symmetric function in the usual sense. A key observation is that it is possible to extend the two classes of symmetric function bases discussed above to paired symmetric functions.

We describe first how to extend the definition of the power-sum basis. If $r \in \mathbb{Z}^{>0}$ and $s \in \mathbb{Z}^{\geq 0}$ define $\bar{p}_{r, s}(\mathbf{x}, \mathbf{t})=\sum_{i=1}^{\infty} x_{i}^{r} t_{i}^{s}$. For an integer pair partition $\bar{\tau}$ let

$$
\bar{p}_{\bar{\tau}}(\mathbf{x}, \mathbf{t})=\prod_{\left(a_{i}, b_{i}\right) \in \bar{\tau}} \bar{p}_{a_{i}, b_{i}}(\mathbf{x}, \mathbf{t}) .
$$

Then the collection $\left\{\bar{p}_{\bar{\tau}}(\mathbf{x}, \mathbf{t}): \bar{\tau} \vdash(n, m)\right\}$ forms a basis for the paired symmetric functions of degrees $n$ and $m$ in respectively $\mathbf{x}$ and $\mathbf{t}$.

A second basis for the paired symmetric functions is defined by extending the definition of the augmented monomial basis. If $\bar{\tau}=\left(\left(a_{1}, b_{1}\right), \ldots,\left(a_{k}, b_{k}\right)\right)$ is an integer pair partition of $(n, m)$ then let

$$
\bar{m}_{\bar{\tau}}(\mathbf{x}, \mathbf{t})=\sum_{i_{1}, \ldots, i_{k}} x_{i_{1}}^{a_{1}}\left(1+t_{i_{1}}\right)^{b_{1}} \cdots x_{i_{k}}^{a_{k}}\left(1+t_{i_{k}}\right)^{b_{k}},
$$

where the summation is over all $k$-tuples $\left(i_{1}, \ldots, i_{k}\right)$ of pairwise distinct strictly positive integers. The collection $\left\{\bar{m}_{\bar{\tau}}(\mathbf{x}, \mathbf{t}): \bar{\tau} \vdash(n, m)\right\}$ forms a basis for the paired symmetric functions of degrees $n$ and $m$ in respectively $\mathbf{x}$ and $\mathbf{t}$.

\section{A menagerie of polynomials 1}

We give definitions of and some relations between a collection of graph polynomials beginning with two very well-established examples and moving on to four that are more recent.

The chromatic polynomial $P_{G}(\lambda)$ was introduced by Birkhoff in 1912 [1] in an effort to prove the four colour theorem. For a positive integer $\lambda$ it is defined

\footnotetext{
${ }^{1}$ The section title is suggested by the title of [9]
} 
to be the number of proper colourings of the vertices of $G$ using colours drawn from a set of size $\lambda$. Whitney [18] showed that

$$
P_{G}(\lambda)=\sum_{A \subseteq E}(-1)^{|A|} \lambda^{k(G \mid A)} .
$$

This relation is one way to prove that the chromatic polynomial is truly a polynomial but more importantly a generalization of it forms the crux of one of our proofs.

Tutte introduced his eponymous polynomial in 15. Given a graph $G$, the Tutte polynomial $T_{G}(x, y)$ is given by

$$
T_{G}(x, y)=\sum_{A \subseteq E}(x-1)^{r(E)-r(A)}(y-1)^{|A|-r(A)} .
$$

Using (3.1) one obtains the well-known specialization

$$
P_{G}(\lambda)=\lambda^{k(G)} T_{G}(1-\lambda, 0) .
$$

Because $k(G)=|V|-r(E)$ and $r(E)$ is determined by the coefficients of the Tutte polynomial we obtain $T \succ P$.

The Tutte polynomial contains a whole host of specializations, for example the number of spanning trees, number of spanning forests and the reliability polynomial as well as applications in statistical mechanics and knot theory. Details of many specializations are contained in 3, 16.

Motivated by a series of papers 4, 5, 6, the weighted graph polynomial $U$ was introduced in [10]. The authors of [4, 5, 6] introduce a graph polynomial derived from Vassiliev invariants of knots and note that this polynomial does not include the Tutte polynomial as a special case. With a slight generalisation of their definition we obtain the weighted graph polynomial $U$ that does include the Tutte polynomial.

The original definition of $U$ involved a recurrence relation using deletion and contraction, but for the purposes of this paper it is most useful to define $U$ using the "states model expansion" from Proposition 5.1 in [10].

$$
U_{G}(\mathbf{x}, y)=U_{G}\left(x_{1}, x_{2}, \ldots, y\right)=\sum_{A \subseteq E} x_{n_{1}} x_{n_{2}} \cdots x_{n_{k(G \mid A)}}(y-1)^{|A|-r(A)},
$$

where $n_{1}, \ldots, n_{k(G \mid A)}$ are the numbers of vertices in the connected components of $G \mid A$ and $x_{1}, x_{2}, \ldots$ are commuting indeterminates. For example, if $G$ is a triangle then

$$
U_{G}(\mathbf{x}, y)=x_{1}^{3}+3 x_{1} x_{2}+3 x_{3}+(y-1) x_{3}=x_{1}^{3}+3 x_{1} x_{2}+2 x_{3}+y x_{3} .
$$

The next few results are all proved in [10]. The first result shows that $U \succ T$.

Proposition 3.3. For any graph $G$,

$$
T_{G}(x, y)=(x-1)^{-k(G)} U_{G}\left(x_{i}=x, y\right) .
$$


Note that we have abused notation somewhat by writing $U_{G}\left(x_{i}=x, y\right)$ where we mean setting $x_{i}=x$ for all $i$.

The attraction of $U$ is that it contains many other graph invariants as specialisations, for instance the 2-polymatroid rank generating function of Oxley and Whittle [11, and as a consequence the matching polynomial and the stable set polynomial 7 .

A stable set in a graph $G$ is a set $S$ of vertices for which $G$ has no edge with both endpoints in $S$. The stability polynomial $A_{G}(p)$ was introduced by Farr in [7] and is given by

$$
A_{G}(p)=\sum_{U \in \mathcal{S}(G)} p^{|U|}(1-p)^{|V(G) \backslash U|},
$$

where $\mathcal{S}(G)$ is the set of all stable sets of $G$.

Proposition 3.4. If $G$ is loopless then $A(G ; p)$ is given by

$$
A_{G}(p)=U_{G}\left(x_{1}=1, x_{j}=-(-p)^{j} \text { for } j \geq 2, y=0\right) .
$$

The two-polymatroid rank generating function $S_{G}(u, v)$ was introduced by Oxley and Whittle in [1] and is defined as follows. Given a graph $G$ and $A \subseteq E(G)$ let $f(A)$ denote the number of vertices of $G$ that are an endpoint of an edge in $A$. Then

$$
S_{G}(u, v)=\sum_{A \subseteq E(G)} u^{|V(G)|-f(A)} v^{2|A|-f(A)} .
$$

$S$ contains the matching polynomial as a specialisation.

Proposition 3.5. Let $G$ be a loopless graph with no isolated vertices. Then

$$
S_{G}(u, v)=U_{G}\left(x_{1}=u, x_{2}=1, x_{j}=v^{j-2} \text { for } j>2, y=v^{2}+1\right) .
$$

The chromatic symmetric function was developed by Stanley in [13. Let $G$ be a graph with vertex set $V=\left\{v_{1}, \ldots, v_{n}\right\}$. Then $X_{G}$ is a homogeneous symmetric function of degree $n$ defined by

$$
X_{G}(\mathbf{x})=X_{G}\left(x_{1}, x_{2}, \ldots\right)=\sum_{\chi} x_{\chi\left(v_{1}\right)} x_{\chi\left(v_{2}\right)} \cdots x_{\chi\left(v_{n}\right)},
$$

where the sum ranges over all proper colourings $\chi: V \rightarrow \mathbb{Z}^{>0}$.

The following result from [10] shows that $U \succ X$.

Proposition 3.6. For any graph $G$

$$
X_{G}(\mathbf{x})=(-1)^{|V|} U_{G}\left(x_{j}=-p_{j}, y=0\right) .
$$


In a second paper on the chromatic symmetric function [14, Stanley introduced the Tutte symmetric function defined by

$$
Y_{G}(\mathbf{x}, t)=Y_{G}\left(x_{1}, x_{2}, \ldots, t\right)=\sum_{\chi} x_{\chi\left(v_{1}\right)} x_{\chi\left(v_{2}\right)} \cdots x_{\chi\left(v_{n}\right)}(1+t)^{b(\chi)},
$$

where the sum is now over all colourings $\chi: V \rightarrow \mathbb{Z}^{>0}$ and $b(\chi)$ is the number of monochromatic edges, that is, edges for which both endpoints receive the same colour.

In [10], the following was shown.

Theorem 3.7. The polynomial $U$ and symmetric function $Y$ are equivalent. In particular $Y_{G}$ is easily obtained from $U_{G}$ by the substitution

$$
Y_{G}(\mathbf{x}, t)=t^{|V|} U_{G}\left(x_{j}=\frac{p_{j}(\mathbf{x})}{t}, y=t+1\right) .
$$

Conversely, if we expand $Y_{G}$ in terms of the power-sum basis then we can recover $U_{G}$.

If $\tau=\left(n_{1}, \ldots, n_{k}\right)$ then we use $\mathbf{x}_{\tau}$ to denote the monomial $\prod_{i=1}^{k} x_{i}^{n_{i}}$. Another way of describing the substitution into $U_{G}$ that produces $Y_{G}$ is to say that if for each $\tau \vdash n$ and each $i$ the monomial $\mathbf{x}_{\tau} y^{i}$ in $U_{G}$ is replaced by $p_{\tau}(\mathbf{x}) t^{n-k(\tau)}(t+1)^{i}$ then $Y_{G}$ is obtained.

The final polynomial that we will define is the polychromate, introduced originally by Brylawski [2. Given a graph $G$ and a partition $\pi$ of its vertices into non-empty blocks, we define $e(\pi)$ to be the number of edges with both endpoints in the same block of the partition.

The polychromate $\chi_{G}(\mathbf{x}, y)$ is defined by

$$
\chi_{G}(\mathbf{x}, y)=\sum_{\pi} y^{e(\pi)} \mathbf{x}_{\tau(\pi)},
$$

where the summation is over all partitions of $V(G)$.

The following result is due to Sarmiento [12].

Theorem 3.8. The polynomials $U$ and $\chi_{G}$ are equivalent.

Obtaining $U$ from $\chi_{G}$ or vice versa is complicated and we do not explain this here but discuss it further at the end of the next section.

\section{Extensions}

\subsection{The extended Tutte symmetric function}

Our extension of Stanley's Tutte symmetric function replaces the $t$ variable by countably infinitely many variables $t_{1}, t_{2}, \ldots$, enumerating not just the total 
number of monochromatic edges but the numbers of monochromatic edges of each colour. It is defined as follows.

$$
\bar{Y}_{G}(\mathbf{x}, \mathbf{t})=\sum_{\chi}\left(\prod_{i=1}^{n} x_{\chi\left(v_{i}\right)}\right)\left(\prod_{i=1}^{\infty}\left(1+t_{i}\right)^{b_{i}(\chi)}\right),
$$

where the sum is over all colourings $\chi: V \rightarrow \mathbb{Z}^{>0}$ and $b_{i}(\chi)$ is the number of monochromatic edges for which both endpoints have colour $i$.

The function $\bar{Y}$ is a paired symmetric function of degrees $n$ and $m$ in respectively the $\mathbf{x}$ and $\mathbf{t}$ variables. Note that $\bar{Y}_{G}$ is not homogenous in $\mathbf{t}$ unless $m=0$.

We can obtain a version of (3.1) which applies to the extended Tutte symmetric function.

Proposition 4.2. For any graph $G$,

$$
\bar{Y}_{G}(\mathbf{x}, \mathbf{t})=\sum_{A \subseteq E} \bar{p}_{\bar{\tau}(\pi(A))}(\mathbf{x}, \mathbf{t}) .
$$

Proof. Given a colouring $\chi$, let $B_{i}(\chi)$ denote the monochromatic edges for which both endpoints have colour $i$. Furthermore let $B(\chi)=\bigcup_{i} B_{i}(\chi)$, the set of all monochromatic edges. For each $i$ we can write

$$
\left(1+t_{i}\right)^{b_{i}(\chi)}=\sum_{A_{i} \subseteq B_{i}(\chi)} t_{i}^{\left|A_{i}\right|} .
$$

So we have

$$
\begin{aligned}
\bar{Y}_{G}(\mathbf{x}, \mathbf{t}) & =\sum_{\chi}\left(\prod_{i=1}^{n} x_{\chi\left(v_{i}\right)}\right)\left(\prod_{i=1}^{\infty} \sum_{A_{i} \subseteq B_{i}(\chi)} t_{i}^{\left|A_{i}\right|}\right) \\
& =\sum_{\chi}\left(\prod_{i=1}^{n} x_{\chi\left(v_{i}\right)}\right) \sum_{A \subseteq B(\chi)}\left(\prod_{i=1}^{\infty} t_{i}^{\left|A \cap B_{i}(\chi)\right|}\right) .
\end{aligned}
$$

By interchanging the order of summation, we obtain

$$
\bar{Y}_{G}(\mathbf{x}, \mathbf{t})=\sum_{A \subseteq E} \sum_{\chi: B(\chi) \supseteq A}\left(\prod_{i=1}^{n} x_{\chi\left(v_{i}\right)}\right)\left(\prod_{i=1}^{\infty} t_{i}^{\left|A \cap B_{i}(\chi)\right|}\right) .
$$

The colourings appearing in the inner summation are precisely those which are monochromatic on the edges of $G \mid A$. So in any such colouring the vertices of a component of $G \mid A$ must all receive the same colour and the colours of the monochromatic edges counted in the final product are determined by the colour of the component of $G \mid A$ to which they belong. Hence for any $A \subseteq E$

$$
\sum_{\chi: B(\chi) \supseteq A}\left(\prod_{i=1}^{n} x_{\chi\left(v_{i}\right)}\right)\left(\prod_{i=1}^{\infty} t_{i}^{\left|A \cap B_{i}(\chi)\right|}\right)=\bar{p}_{\bar{\tau}(\pi(A))}(\mathbf{x}, \mathbf{t})
$$

and the result follows. 


\subsection{The extended U-polynomial}

The extended $U$ polynomial, which we denote by $\bar{U}$, is a polynomial in countably many commuting variables $z_{i, j}$ where $i \in \mathbb{Z}^{>0}$ and $j \in \mathbb{Z}^{\geq 0}$. The definition is a natural generalization of (3.2) and was first introduced in [17.

$$
\bar{U}_{G}(\mathbf{z})=\sum_{A \subseteq E} z_{c_{1}, e_{1}-c_{1}+1} z_{c_{2}, e_{2}-c_{2}+1} \cdots z_{c_{k(G \mid A)}, e_{k(G \mid A)}-c_{k(G \mid A)}+1},
$$

where $c_{i}$ and $e_{i}$ are respectively the number of vertices and edges in the $i$ th connected component of $G \mid A$.

For example if $G$ is a triangle then

$$
\bar{U}_{G}(\mathbf{z})=\left(z_{1,0}\right)^{3}+3 z_{1,0} z_{2,0}+3 z_{3,0}+z_{3,1} .
$$

Observe that

$$
U_{G}(\mathbf{x}, y)=\bar{U}_{G}\left(z_{i j}=x_{i}(y-1)^{j}\right)
$$

and so $\bar{U} \succ U$. If we take $G_{1}\left(G_{2}\right)$ to be a path of length two with a loop attached at a vertex of degree one (two) then

$$
U_{G_{1}}(\mathbf{x}, y)=U_{G_{2}}(\mathbf{x}, y)=y\left(x_{3}+2 x_{2} x_{1}+x_{1}^{3}\right) .
$$

However

$$
\bar{U}_{G_{1}}(\mathbf{z})=z_{3,1}+z_{3,0}+z_{2,1} z_{1,0}+z_{2,0} z_{1,1}+2 z_{2,0} z_{1,0}+z_{1,0}^{2} z_{1,1}+z_{1,0}^{3}
$$

but

$$
\bar{U}_{G_{2}}(\mathbf{z})=z_{3,1}+z_{3,0}+2 z_{2,1} z_{1,0}+2 z_{2,0} z_{1,0}+z_{1,0}^{2} z_{1,1}+z_{1,0}^{3} .
$$

Unfortunately we do not know of a pair of loopless graphs for which $U_{G_{1}}=U_{G_{2}}$ but $\bar{U}_{G_{1}} \neq \bar{U}_{G_{2}}$.

We now show that $\bar{U}$ and $\bar{Y}$ are equivalent.

Theorem 4.4. The polynomial $\bar{U}$ and the extended Tutte symmetric function are equivalent. More precisely

$$
\bar{Y}_{G}(\mathbf{x}, \mathbf{t})=\bar{U}_{G}\left(z_{i, j}=\bar{p}_{i, i+j-1}(\mathbf{x}, \mathbf{t})\right) .
$$

Furthermore if we express $\bar{Y}_{G}$ in terms of the power-sum basis as

$$
\bar{Y}_{G}(\mathbf{x}, \mathbf{t})=\sum_{\bar{\tau} \vdash(n, m)} a_{\bar{\tau}} \bar{p}_{\bar{\tau}}(\mathbf{x}, \mathbf{t}),
$$

we obtain $\bar{U}_{G}$ by replacing $\bar{p}_{r_{1}, s_{1}} \cdots \bar{p}_{r_{k}, s_{k}}$ by $z_{r_{1}, s_{1}-r_{1}+1} \cdots z_{r_{k}, s_{k}-r_{k}+1}$.

Proof. The result follows easily from Proposition 4.2, Note that

$$
\bar{Y}_{G}(\mathbf{x}, \mathbf{t})=\sum_{A \subseteq E} \bar{p}_{\bar{\tau}(\pi(A))}(\mathbf{x}, \mathbf{t})=\sum_{A \subseteq E} \bar{p}_{c_{1}, e_{1}} \cdots \bar{p}_{c_{k}(G \mid A), e_{k}(G \mid A)},
$$

where $c_{i}, e_{i}$ are respectively the number of vertices and number of edges in the $i$ th component of $G \mid A$. Comparing this expression with (4.3) gives the result. 
There is a recurrence relation for the extended $U$-polynomial involving deletion and contraction just as there is for $U$ itself [10. The recurrence relation for $U$ involves a more general polynomial $W$ defined on graphs where the vertices have strictly positive integer weights. In order to describe the recurrence for the extended $U$-polynomial we need to define an extended version of $W$. We use the notation $(G, \omega)$ to describe a graph $G$ with a strictly positive integer weight $\omega(v)$ attached at each vertex $v$. We then let $\bar{W}_{(G, \omega)}(\mathbf{z})$ be a polynomial in countably many commuting variables $z_{i, j}$ where $i \in \mathbb{Z}^{>0}$ and $j \in \mathbb{Z}^{\geq 0}$ and be given by

$$
\bar{W}_{(G, \omega)}(\mathbf{z})=\sum_{A \subseteq E} z_{w_{1}, e_{1}-c_{1}+1} z_{w_{2}, e_{2}-c_{2}+1} \cdots z_{w_{k(G \mid A)}, e_{k(G \mid A)}-c_{k(G \mid A)}+1},
$$

where $c_{i}, e_{i}$ and $w_{i}$ are respectively the number of vertices, the number of edges and the sum of the weights on the vertices in the $i$ th connected component of $G \mid A$.

For example if $(G, \omega)$ is a triangle for which the vertices have weights $a, b$ and $c$ then

$\bar{W}_{(G, \omega)}(\mathbf{z})=z_{a, 0} z_{b, 0} z_{c, 0}+z_{a, 0} z_{b+c, 0}+z_{b, 0} z_{c+a, 0}+z_{c, 0} z_{a+b, 0}+3 z_{a+b+c, 0}+z_{a+b+c, 1}$.

If we set $\omega(v)=1$ for all $v$ then in (4.5) we have $w_{i}=c_{i}$ for each $i$ and we obtain $\bar{U}_{G}(\mathbf{z})=\bar{W}_{(G, \omega)}(\mathbf{z})$.

We now define deletion and contraction of edges in a weighted graph. For any edge $e$ of a weighted graph $(G, \omega)$, the deletion of $e$, denoted by $(G, \omega)-e$ is formed by removing $e$ from $E(G)$. For a non-loop edge $e$ with endpoints $u$ and $v$, the contraction of $e$, denoted by $(G, \omega) / e$ is formed by removing $e$ from $E(G)$ and identifying the vertices $u$ and $v$ to form a new vertex $w$ having weight $\omega(u)+\omega(v)$. So both operations conserve the total weight of the vertices.

\section{Theorem 4.6.}

1. Suppose that the only edges of $(G, \omega)$ are loops. Let $V=\left\{v_{1}, \ldots, v_{n}\right\}$ and suppose that $\omega\left(v_{i}\right)=w_{i}$ and that there are $e_{i}$ loops attached at $v_{i}$. Then

$$
\bar{W}_{(G, \omega)}=\prod_{i=1}^{n} \sum_{j=0}^{e_{i}}\left(\begin{array}{c}
e_{i} \\
j
\end{array}\right) z_{w_{i}, j} .
$$

2. If $e$ is an edge of $(G, \omega)$ that is not a loop then

$$
\bar{W}_{(G, \omega)}=\bar{W}_{(G, \omega)-e}+\bar{W}_{(G, \omega) / e} .
$$

Proof. The first part follows immediately from the definition. To prove the second part, suppose that $e$ is an edge of $(G, \omega)$ that is not a loop. By splitting the sum in the definition of $\bar{W}$ depending on whether or not $A$ contains $e$ we 
obtain

$$
\begin{aligned}
\bar{W}_{(G, \omega)}(\mathbf{z})= & \sum_{A \subseteq E} z_{w_{1}, e_{1}-c_{1}+1} \cdots z_{w_{k(G \mid A)}, e_{k(G \mid A)}-c_{k(G \mid A)}+1} \\
= & \sum_{A \subseteq E-e} z_{w_{1}, e_{1}-c_{1}+1} \cdots z_{w_{k(G \mid A)}, e_{k(G \mid A)}-c_{k(G \mid A)}+1} \\
& +\sum_{e \in A \subseteq E} z_{w_{1}, e_{1}-c_{1}+1} \cdots z_{w_{k(G \mid A)}, e_{k(G \mid A)}-c_{k(G \mid A)}+1} .
\end{aligned}
$$

From now on we just write $G$ rather than $(G, \omega)$. The first term is $\bar{W}_{G-e}(\mathbf{z})$ and we claim that the second term is $\bar{W}_{G / e}(\mathbf{z})$. To show this we compare the terms appearing in the second sum in (4.9) with those in the definition of $\bar{W}$ applied to $G / e$. Let $A \subseteq E-e$. Compare the connected components of $G \mid(A \cup e)$ and $(G / e) \mid A$. One component $C$ of $G \mid(A \cup e)$ contains $e$. Suppose the endpoints of $e$ are $v$ and $w$. Then there is a component of $(G / e) \mid A$ for which the vertices are those of $C-\{v, w\}$ together with the new vertex formed when $e$ was contracted. The weight of the new vertex is $\omega(v)+\omega(w)$ and the weight of all the other vertices in $C$ is the same in $G \mid(A \cup e)$ as in $(G / e) \mid A$ so the total weight of the component is unchanged. Since $e$ has been removed there is one more edge in this component in $G \mid(A \cup e)$ compared with $(G / e) \mid A$. Similarly there is one more vertex in the is component in $G \mid(A \cup e)$ compared with $(G / e) \mid A$. Every other component other than $C$ has the same vertices with the same weights and the same edges in both $G \mid(A \cup e)$ and $(G / e) \mid A$. Hence the terms appearing in the second sum in (4.9) are exactly those appearing in $\bar{W}_{G / e}(\mathbf{z})$ and so the claim and hence the theorem are proved.

To illustrate this theorem we show how to compute $\bar{U}$ for the following graph.

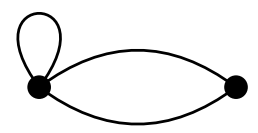

To do this we add weight one to each vertex and compute $\bar{W}$ of the corresponding weighted graph. We use the convention that a depiction of a graph means $\bar{W}$ of that graph.

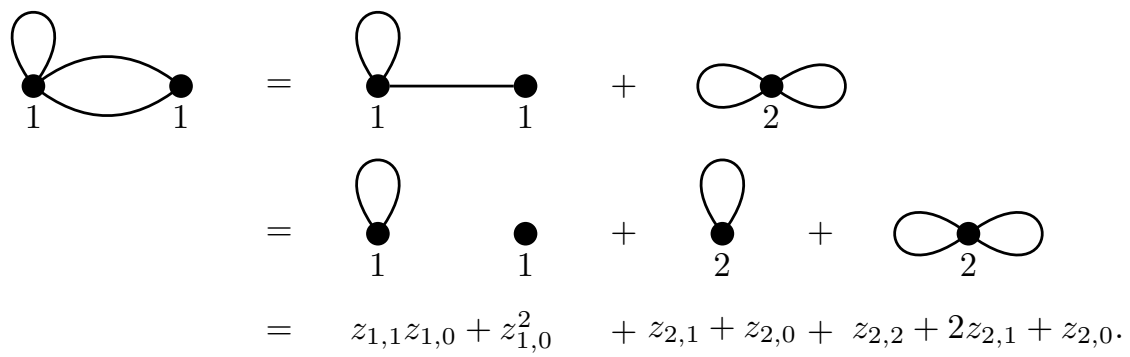


We can now justify our claim in the introduction that the extended $U$ polynomial (and as a corollary of the other results in this paper the extended polychromate and extended Tutte symmetric function) specializes to Tutte's universal $V$-functions.

The universal $V$-function is a polynomial in the commuting indeterminates $\mathbf{y}=\left(y_{0}, \ldots, y_{m}\right)$ and is defined recursively as follows. If the only edges of $G$ are loops and the number of loops on the vertices are $e_{1}, \ldots, e_{n}$ then

$$
V_{G}(\mathbf{y})=\prod_{i=1}^{n} y_{e_{i}}
$$

Otherwise for any edge $e$ that is not a loop

$$
V_{G}(\mathbf{y})=V_{G-e}(\mathbf{y})+V_{G / e}(\mathbf{y}) .
$$

It is relatively simple to prove by induction that the definition is independent of the choice of edge in (4.10).

\section{Proposition 4.11.}

$$
V_{G}(\mathbf{y})=\bar{U}_{G}\left(z_{i j}=\sum_{k=0}^{j}(-1)^{j-k}\left(\begin{array}{l}
j \\
k
\end{array}\right) y_{k}\right) .
$$

Proof. Notice that it follows from (4.5) that if the value of $z_{i j}$ does not depend on $i$ then for any $\omega$ and $\omega^{\prime}, \bar{W}_{(G, \omega)}(\mathbf{z})=\bar{W}_{\left(G, \omega^{\prime}\right)}(\mathbf{z})$. In particular if for all $v$, $\omega^{\prime}(v)=1$, we get $\bar{W}_{(G, \omega)}(\mathbf{z})=\bar{U}_{G}(\mathbf{z})$. So $\bar{U}_{G}\left(z_{i j}=\sum_{k=0}^{j}(-1)^{j-k}\left(\begin{array}{l}j \\ k\end{array}\right) y_{k}\right)$ must satisfy (4.7) and (4.8). It follows from (4.8) that $\bar{U}_{G}\left(z_{i j}=\sum_{k=0}^{j}(-1)^{j-k}\left(\begin{array}{l}j \\ k\end{array}\right) y_{k}\right)$ satisfies (4.10). From (4.7), we see that if the only edges of $G$ are loops and the number of loops on the vertices are $e_{1}, \ldots, e_{n}$ then

$$
\begin{aligned}
\bar{U}_{G}\left(z_{i j}=\sum_{k=0}^{j}(-1)^{j-k}\left(\begin{array}{l}
j \\
k
\end{array}\right) y_{k}\right) & =\prod_{i=1}^{n} \sum_{j=0}^{e_{i}}\left(\begin{array}{c}
e_{i} \\
j
\end{array}\right) \sum_{k=0}^{j}(-1)^{j-k}\left(\begin{array}{l}
j \\
k
\end{array}\right) y_{k} \\
& =\prod_{i=1}^{n} y_{e_{i}} .
\end{aligned}
$$

\subsection{The extended polychromate}

Like the extended $U$-polynomial, the extended polychromate is a polynomial in countably infinitely many commuting variables $x_{i, j}$ where $i \in \mathbb{Z}^{>0}$ and $j \in \mathbb{Z}^{\geq 0}$. It was also first introduced in [17.

The extended polychromate $\bar{\chi}$ is defined as follows.

$$
\bar{\chi}_{G}(\mathbf{x})=\sum_{\pi} \overline{\mathbf{x}}(\bar{\tau}(\pi))
$$


where the sum is over all partitions of $V$ and if $\bar{\tau}=\left(\left(a_{1}, b_{1}\right), \ldots,\left(a_{k}, b_{k}\right)\right)$ then $\overline{\mathbf{x}}(\bar{\tau})=x_{a_{1}, b_{1}} \cdots x_{a_{k}, b_{k}}$.

For example if $G$ is a triangle then

$$
\bar{\chi}_{G}(\mathbf{x})=x_{1,0}^{3}+3 x_{2,1} x_{1,0}+x_{3,3}
$$

and if $G$ is a path with two edges then

$$
\bar{\chi}_{G}(\mathbf{x})=x_{1,0}^{3}+2 x_{2,1} x_{1,0}+x_{2,0} x_{1,0}+x_{3,2} .
$$

Note that we obtain the polychromate by substituting $x_{i, j}=x_{i} y^{j}$ resulting in a polynomial in $x_{1}, \ldots, x_{n}$ and $y$.

We now show that the extended polychromate and the extended Tutte symmetric function are equivalent

Theorem 4.12. The extended polychromate and the extended Tutte symmetric function are equivalent. More precisely for each $\bar{\tau}=\left(\left(a_{1}, b_{1}\right), \ldots,\left(a_{k}, b_{k}\right)\right) \vdash$ $(n, m)$, the coefficient of $\bar{m}_{\bar{\tau}}(\mathbf{x}, \mathbf{t})$ in $\bar{Y}_{G}$ is the same as the coefficient of $\overline{\mathbf{x}}_{\bar{\tau}}$ in $\bar{\chi}_{G}$.

Proof. A colouring of $G$ induces a partition of $V$ in which two vertices are in the same block if and only if they receive the same colour. So we may partition the sum in (4.1) according to the partition of $V$ induced by the colouring. Hence we can write

$$
\bar{Y}_{G}(\mathbf{x} ; \mathbf{t})=\sum_{\pi} \sum_{\chi}\left(\prod_{i=1}^{n} x_{\chi\left(v_{i}\right)}\right)\left(\prod_{i=1}^{\infty}\left(1+t_{i}\right)^{b_{i}(\chi)}\right),
$$

where the first summation is over all partitions of $V$ and the second over all colourings of $V$ with strictly positive integers so that vertices receive the same colour if and only if they are in the same block of $\pi$. Fix a partition $\pi$ of $V$ and suppose that $\bar{\tau}(\pi)=\left(\left(a_{1}, b_{1}\right), \ldots,\left(a_{k}, b_{k}\right)\right)$. Then the contribution to $\bar{Y}$ from colourings inducing $\pi$ is $\bar{m}_{\bar{\tau}}(\mathbf{x}, \mathbf{t})$. However the monomial in $\bar{\chi}_{G}$ corresponding to $\pi$ is $\overline{\mathbf{x}}(\bar{\tau})$ and the result follows.

Corollary 4.13. The extended polychromate and the extended $U$-polynomial are equivalent.

Proof. This follows easily from the transitivity of equivalence.

In principle one could describe a substitution in order to obtain $\bar{\chi}$ from $\bar{U}$ or vice versa but the procedure would be very complicated. We show briefly how Sarmiento's result from 12 may be obtained as a special case of our results by comparing the expressions linking the Tutte symmetric function with the $U$-polynomial in Theorem 3.7 and an expression linking the Tutte symmetric function with the polychromate deduced from Theorem 4.12

Recall that the symmetric Tutte function is a function of $\left(x_{1}, x_{2}, \ldots, t\right)$ and is a homogenous symmetric function of degree $n$ in the $\mathbf{x}$ variables. Furthermore 
recall that both the collections $\left\{p_{\tau}(\mathbf{x}): \tau \vdash n\right\}$ and $\left\{m_{\tau}(\mathbf{x}): \tau \vdash n\right\}$ are bases for the homogenous symmetric functions of degree $n$ in $\mathbf{x}$. Consequently there are constants $a_{\tau, \tau^{\prime}}$ such that $p_{\tau}(\mathbf{x})=\sum_{\tau^{\prime}} a_{\tau, \tau^{\prime}} m_{\tau^{\prime}}(\mathbf{x})$.

It is not difficult to compute $a_{\tau, \tau^{\prime}}$. Given a partition $\pi$, we say that the partition $\pi^{\prime}$ is a coarsening of $\pi$ if every block of $\pi^{\prime}$ is a union of blocks of $\pi$. Let $\pi$ be a partition of $\{1, \ldots, n\}$ of type $\tau$. Then $a_{\tau, \tau^{\prime}}$ is the number of coarsenings of $\pi$ of type $\tau^{\prime}$.

Proposition 4.14. The polychromate may be obtained from the U-polynomial by replacing for each $\tau$ such that $\tau \vdash n$, the monomial $\mathbf{x}_{\tau} y^{j}$ by $\sum_{\tau^{\prime}} a_{\tau, \tau^{\prime}} \mathbf{x}_{\tau^{\prime}} y^{j}(y-$ $1)^{n-k(\tau)}$ where the sum is over all $\tau^{\prime} \vdash n$.

Proof. Setting $t_{i}=t$ for all $i$ in the extended symmetric Tutte function we can write

$$
Y_{G}(\mathbf{x}, t)=\sum_{\tau \vdash n} \sum_{i} c_{\tau, i} m_{\tau}(\mathbf{x})(1+t)^{i}
$$

for certain constants $c_{\tau, i}$. Recall that if $\tau=\left(n_{1}, \ldots, n_{k}\right)$ then $x_{\tau}=x_{n_{1}} \cdots x_{n_{k}}$. The polychromate may be written in the form

$$
\chi_{G}(\mathbf{x}, t)=\sum_{\tau \vdash n} \sum_{i} c_{\tau, i}^{\prime} \mathbf{x}_{\tau} t^{i},
$$

for certain constants $c_{\tau, i}^{\prime}$. Theorem 4.12 implies that for all $\tau$ and $i, c_{\tau, i}=c_{\tau, i}^{\prime}$.

The remarks immediately after Theorem 3.7 state that $Y_{G}$ may be obtained from $U_{G}$ by replacing the monomial $\mathbf{x}_{\tau} y^{i}$ in $U_{G}$ by $p_{\tau}(\mathbf{x}) t^{n-k(\tau)}(t+1)^{i}$. Given the relationship between the the power-sum basis and the augmented monomial basis an equivalent substitution is to replace $\mathbf{x}_{\tau} y^{i}$ by $t^{n-k(\tau)}(t+$ $1)^{i} \sum_{\tau^{\prime} \vdash n} a_{\tau, \tau^{\prime}} m_{\tau^{\prime}}(\mathbf{x})$.

Now the first part of the proof shows that replacing $t$ by $y-1$ and $m_{\tau}(\mathbf{x})$ by $\mathbf{x}_{\tau}$ in $Y_{G}$ gives $\chi_{G}$ and the result follows.

A similar argument shows how to obtain $U$ from the polychromate.

\section{Conclusions and open problems}

The graph polynomials and symmetric functions that we have discussed are related by the following partial order where a function $P$ is above $Q$ if $P$ specializes to $Q$. 


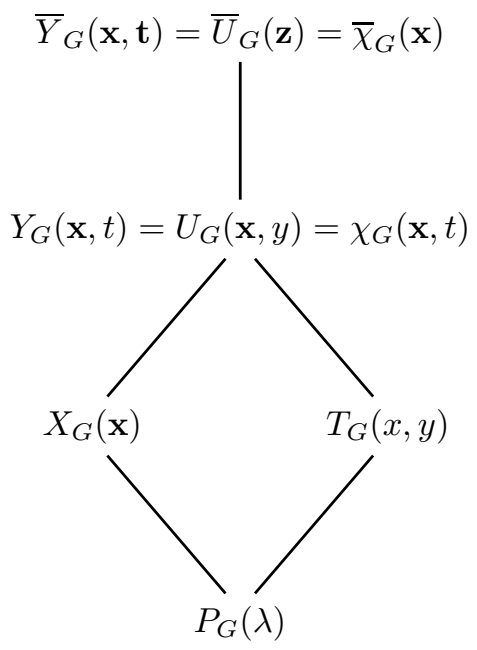

The relationships between many other polynomials are considered in 9 .

An open problem is to find a pair of loopless graphs $G_{1}, G_{2}$ for which $U_{G_{1}}=$ $U_{G_{2}}$ (or for which either of the other equivalent functions coincide) but $\bar{U}_{G_{1}} \neq$ $\bar{U}_{G_{2}}$. The following graphs are the smallest known pair of non-isomorphic graphs with the same polychromate 2]. However it is easy to see that they also have the same extended $U$-polynomial.
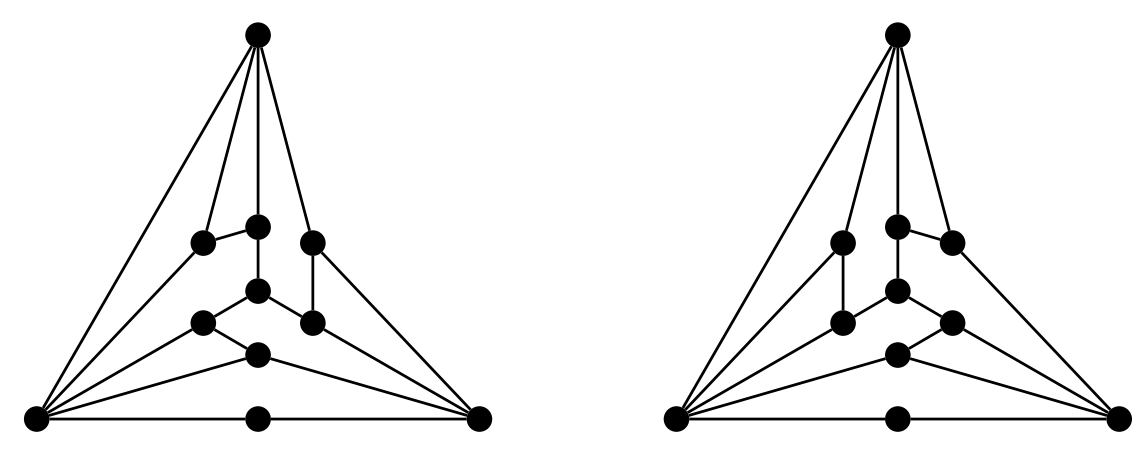

\section{Acknowledgements}

We would like to thank Dominic Welsh and Janos Makowsky for useful discussions. 


\section{References}

[1] G. D. Birkhoff. A determinant formula for the number of ways of coloring a map. Annals of Mathematics, 14:42-46, 1912.

[2] T. H. Brylawski. Intersection theory for graphs. Journal of Combinatorial Theory Series B, 30:233-246, 1981.

[3] T. H. Brylawski and J. G. Oxley. The Tutte polynomial and its applications. In N. White, editor, Matroid applications, pages 123-225. Cambridge University Press, Cambridge, 1992.

[4] S. V. Chmutov, S. V. Duzhin, and S. K. Lando. Vassiliev knot invariants: I. Introduction. Advances in Soviet Mathematics, 21:117-126, 1994.

[5] S. V. Chmutov, S. V. Duzhin, and S. K. Lando. Vassiliev knot invariants: II. Intersection graph for trees. Advances in Soviet Mathematics, 21:127$134,1994$.

[6] S. V. Chmutov, S. V. Duzhin, and S. K. Lando. Vassiliev knot invariants: III. Forest algebra and weighted graphs. Advances in Soviet Mathematics, 21:135-145, 1994.

[7] G. E. Farr. A correlation inequality involving stable sets and chromatic polynomials. Journal of Combinatorial Theory Series B, 58(1):14-21, 1993.

[8] I. G. MacDonald. Symmetric functions and Hall polynomials. Oxford Mathematical Monographs. Oxford University Press, New York, 1979.

[9] J. A. Makowsky. From a zoo to a zoology: towards a general theory of graph polynomials. To appear in Theory of Computing Systems.

[10] S. D. Noble and D. J. A. Welsh. A weighted graph polynomial from chromatic invariants of knots. Annales de l'institut Fourier, 49(3):1057-1087, 1999.

[11] J. G. Oxley and G. P. Whittle. Tutte invariants for 2-polymatroids. In N. Robertson and P. D. Seymour, editors, Graph Structure Theory, number 147 in Contemporary Mathematics, pages 9-19. AMS, 1993.

[12] I. Sarmiento. The polychromate and a chord diagram polynomial. Annals of Combinatorics, 4:227-236, 2000.

[13] R. P. Stanley. A symmetric function generalisation of the chromatic polynomial of a graph. Advances in Mathematics, 111(1):166-194, March 1995.

[14] R. P. Stanley. Graph colourings and related symmetric functions: ideas and applications. A description of results, interesting applications, \& notable open problems. Discrete Mathematics, 193(1-3):267-286, 1998. 
[15] W. T. Tutte. A ring in graph theory. Mathematical Proceedings of the Cambridge Philosophical Society, 43:26-40, 1947.

[16] D. J. A. Welsh. Complexity : Knots, Colourings, and Counting. Number 186 in London Mathematical Society Lecture Note Series. Cambridge University Press, Cambridge, 1993.

[17] D. J. A. Welsh. Graph polynomials: some questions. Talk at Annual One-Day Combinatorics Colloquium, Reading, 2005.

[18] H. Whitney. The coloring of graphs. Annals of Mathematics, 33:688-718, 1932. 